This item was submitted to Loughborough's Research Repository by the author.

Items in Figshare are protected by copyright, with all rights reserved, unless otherwise indicated.

\title{
Dynamic modeling and control system design for tri-rotor UAV
}

PLEASE CITE THE PUBLISHED VERSION

http://dx.doi.org/10.1109/ISSCAA.2010.5632868

PUBLISHER

(c) IEEE

VERSION

AM (Accepted Manuscript)

LICENCE

CC BY-NC-ND 4.0

REPOSITORY RECORD

Yoo, Dong-Wan, Hyondong Oh, Dae-Yeon Won, and Min-Jea Tahk. 2015. "Dynamic Modeling and Control System Design for Tri-rotor UAV". figshare. https://hdl.handle.net/2134/17851. 


\title{
Dynamic Modeling and Control System Design for Tri-rotor UAV
}

\author{
Dong-Wan Yoo, Hyon-Dong Oh, Dae-Yeon Won, and Min-Jea Tahk
}

Department of Aerospace Engineering, Korea Advanced Institute of Science and Technology

\begin{abstract}
In this paper, design, dynamics and control allocation of Tri-Rotor UAV are introduced. Tri-Rotor UAV has three rotor axes that are equidistant from its center of gravity. There are two designs of Tri-Rotor introduced in this paper. Single Tri-Rotor UAV has a servo-motor that has been installed on one of the three rotors, which enables a rapid control on its motion and its various attitude changes; unlike a Quad-Rotor UAV, which only depends on rpm of four rotors to control. The other design is called Coaxial Tri-Rotor UAV, which has two rotors installed on each rotor axis. Since Tri-Rotor type UAV has the yawing problem induced from an unpaired rotor's reaction torque, it is required to derive accurate dynamics and design control logics for both Single and Coaxial Tri-Rotors. For that reason, control strategy for each Tri-Rotor type is proposed and nonlinear simulations of altitude, Euler angles, and angular velocity responses have been done by using classical PID controller. Simulation results show that the proposed control strategies are appropriate for the control of Single and Coaxial Tri-Rotor UAV.
\end{abstract}

\section{INTRODUCTION}

$\mathrm{T}$ There are some difficult situations, which one could not do his job properly, such as working in steep terrain, disasters, military operational areas, or small indoor facilities. Since such areas have limitations of having massive equipments to operate a mission, there needs to be something that could solve such problems. In order to help work in such difficult situations, unmanned aerial vehicles (UAVs) have been thought as the solution, and many researches relating to this topic are still in process today. There are some requirements for UAVs used in such areas. First, the UAV should be small sized in order to guarantee its free motions at any circumstances. Second, the UAV needs to have quick movements so that it could avoid a sudden collision. Finally, the UAV requires having availabilities in vertical takeoff and landing, and also in sudden directional turns. To satisfy all of those requirements, a multi-rotor thrust UAV is considered to be the best solution for this. Multi-rotor type UAV has

Manuscript received November 30, 2009.

This work was supported in part by Agency for Defense Development in Korea under the grant ADD and by UTRC and BK 21 Project, KAIST

D. W. Yoo is MS student of the Dept. of Aerospace Eng., Korea Advanced Institute of Science and Technology (KAIST), Daejeon, Korea (corresponding author to provide phone: +82-42-350-3794; fax +82-42-350-3702; e-mail: dwyoo@ fdcl.kaist.ac.kr).

H. D. Oh is MS student of the Dept. of Aerospace Eng., KAIST (e-mail : hdoh@fdcl.kaist.ac.kr)

D. Y. Won is Ph.D. Candidate of the Dept. of Aerospace Eng., KAIST (e-mail : dywon@fdcl.kaist.ac.kr).

M. J. Tahk is professor of the Dept. of Aerospace Eng., KAIST (e-mail : mjtahk@fdcl.kaist.ac.kr) various kinds including Double-rotor, Tri-Rotor, Quad-rotor, and also the conventional helicopter type [1-2]. Out of these types of designs, Tri-Rotor, which has three axes of rotation forming a triangular shape, is the main concern of this paper.

Tri-Rotor UAV has a problem of the yawing moment induced by the reaction torque from the unpaired rotor. In order to solve this problem, several designs of Tri-Rotor UAV have their own solutions. First type is called Single Tri-Rotor, and its research has already been done in Compiegne UTC [3]. The main idea of this type is that a servo motor is installed on one of its rotors, which gets tilted with some angle to cancel the yawing moment [4]. The main advantage of having this design is a better movement, especially a quicker turn, from the tilting the one of the rotor axis [5]. The other type of Tri-Rotor UAV is called Coaxial Tri-Rotor, and Draganflyer X6 from Draganfly Innovation Inc. could be considered as this type. It has two rotors installed on each rotor axis, so it has six rotors total. By having two counter rotating rotors on each axis, the reaction torque has naturally been cancelled. The advantage of this type is having a better stability than the Single Tri-Rotor UAV type.

In this paper, small sized Tri-Rotor UAV with vertical takeoff and landing ability has been considered and described In order to apply Tri-Rotor concept into real world flying UAVs, hardware testings for both types of Tri-Rotor are necessary. Before the experimental testing step, numerical simulation for nonlinear system of UAVs should be done. Thus, control strategy for each Tri-Rotor type is proposed and nonlinear simulations of altitude, Euler angles, and angular velocity responses have been done by using classical PID controller. This paper consists as follows. In section 2, the rigid body equations of motion of Tri-Rotor UAV are explained. Then, the control strategies of both Single and Coaxial Tri-Rotors are introduced as well as their force and moment equations. Control system designs for both TriRotors are introduced in section 4, and finally, nonlinear simulation results for altitude and attitude control are represented.

\section{RIGID BODY EQUATIONS OF MOTION}

Considering the size of Tri-Rotor UAVs relative to the surroundings, Tri-Rotor UAVs were assumed to be as rigid objects. The developments of the 6-DOF nonlinear equations of motion for conventional UAVs are also used for Tri-Rotor UAVs. Tri-Rotor UAV is free to rotate and translate in 6-DOF, and the rigid body dynamics are derived by the Newton's Laws [6-7]. For Tri-Rotor UAV, 6-DOF rigid- 
body equations of motions are expressed as the differential equations describing the translational motion, rotational motion, and kinematics as given:

\section{Force Equations}

$$
\begin{aligned}
& \dot{u}=r v-q w-g \sin \theta+F_{X} / m \\
& \dot{v}=-r u+p w+g \sin \phi \cos \theta+F_{Y} / m \\
& \dot{w}=q u-p v+g \cos \phi \cos \theta+F_{Z} / m
\end{aligned}
$$

\section{Moment Equations}

$$
\begin{aligned}
& \dot{p}=\left(I_{y y}-I_{z z}\right) q r / I_{x x}+L / I_{x x} \\
& \dot{q}=\left(I_{z z}-I_{x x}\right) p r / I_{y y}+M / I_{y y} \\
& \dot{r}=\left(I_{x x}-I_{y y}\right) p q / I_{z z}+N / I_{z z}
\end{aligned}
$$

\section{$\underline{\text { Kinematic Equations }}$}

$$
\begin{aligned}
& \dot{\phi}=p+q \sin \phi \tan \theta+r \cos \phi \tan \theta \\
& \dot{\theta}=q \cos \phi-r \sin \phi \\
& \dot{\psi}=(q \sin \phi+r \cos \phi) \sec \theta
\end{aligned}
$$

where $\left(F_{x}, F_{y}, F_{z}\right)$ are the external forces, and $(L, M, N)$ are the external moments acting on the center of gravity with respect to the body-fixed frame. $(u, v, w)$ are translational velocities, $(p, q, r)$ are the rotational velocities, and $(\varphi, \theta, \psi)$ are the rotational angles. $I_{x x}, I_{y y}$, and $I_{z z}$ are the rotational inertia or Tri-Rotor UAV.

\section{SingLE TRI-Rotor UAV}

Single Tri-Rotor has three rotors, and one of the rotors, tail rotor to be specific, is tilted to get rid of the reaction torque problem. Single Tri-Rotor has an advantage of having a rapid motion generated from its tilt rotor, which could also be the defect of this system since it requires a very accurate value of tilting angle for stabilization of the system.

\section{A. Concepts and Dynamics}

Figure 1 shows the configuration of single Tri-Rotor UAV with Reference and body frames.

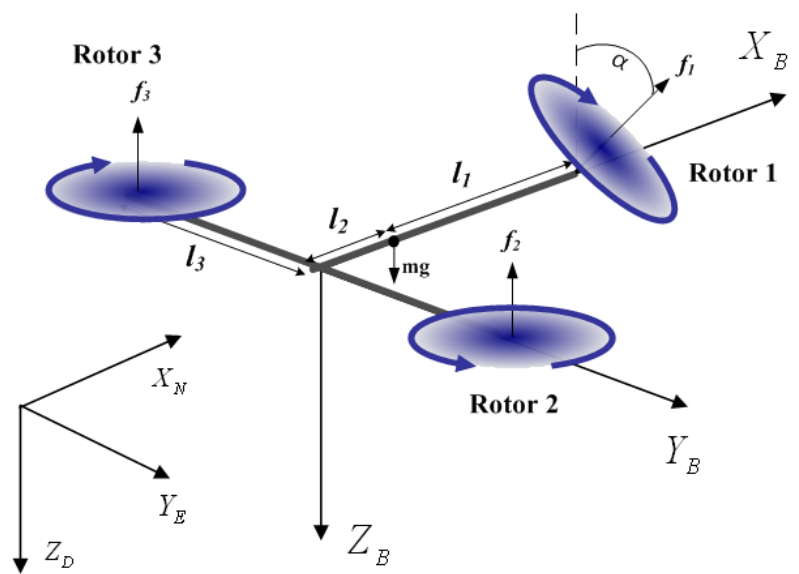

Fig. 1. Configuration of Tri-Rotor UAV with reference and body frames
As shown in figure 1 , the distances $l_{1}, l_{2}, l_{3}$ are defined, and the distances from the center of gravity to each rotor are identical. Two front rotors, rotor 2 and 3, rotate in other directions, and the tilting rotor, rotor 1 , rotates in the same direction as rotor 3 . Therefore, without tilting the rotor 1 , the whole system tend to have a yawing moment in counter clock wise direction, and this is the reason for having a servo motor on the rotor 1 axis for tilting purpose. As the rotor 1 tilts as shown in figure 1 , it creates a moment that cancels the yawing moment of the system. A very accurate calculation of tilting angle $\alpha$ is essential for this system since it has the role for motion controls as well as hovering control. Forces and moments are derived with respect to figure 1 . Forces and moments equations of Single Tri-Rotor are expressed in equations (4) and (5).

$$
\begin{gathered}
\vec{F}=\left[\begin{array}{c}
0 \\
f_{1} \sin \alpha \\
-f_{2}-f_{3}-f_{1} \cos \alpha
\end{array}\right] \\
\vec{M}=\left[\begin{array}{c}
-l_{3}\left(f_{2}-f_{3}\right) \\
-l_{2}\left(f_{2}+f_{3}\right)+l_{1} f_{1} \cos \alpha \\
l_{1} f_{1} \sin \alpha-\tau_{1}+\tau_{2}-\tau_{3}
\end{array}\right]
\end{gathered}
$$

$\alpha$ is the tilt angle of rotor 1 as shown in figure 1. $f_{1}, f_{2}, f_{3}$ are rotor forces and $\tau_{1}, \tau_{2}, \tau_{3}$ are rotor torques, and they are defined in equation (6). $\mathrm{K}_{\mathrm{t}}$ and $\mathrm{K}_{\tau}$ are thrust and torque coefficients, and $\Omega$ is the angular velocity of the rotor.

$$
\begin{aligned}
& f_{i}=k_{t} \cdot \Omega_{i}^{2} \\
& \tau_{i}=k_{\tau} \cdot \Omega_{i}^{2}
\end{aligned}
$$

\section{B. Control Strategies}

As it was mentioned above, tilting angle $\alpha$ has an important role on control of the Single Tri-Rotor. Tri-Rotor's motions could be divided into altitude, roll, pitch, and yaw controls. Control strategies of Single Tri-Rotors are shown in figure 2. Figure 2(a) shows the altitude control, and it shows increasing rotor speeds of each rotor will increase the altitude, and vice versa. Figure 2(b) shows the roll control, and the way of controlling is that given the same rotor 1 speed, varying the rotor speeds from front two rotors will generate a roll control. Figure 2(c) shows the pitch control, and given the same front two rotor's angular velocities, varying the rotor speed of rotor 1 will generate the pitch control. For the yaw control, by using its natural yawing moment from reaction torque and also from the tilt angle $\alpha$, yaw control could successfully generated. Tilt angle $\alpha$ is very useful when encountering a sudden danger of collision because by tilting the rotor, a sudden turning control would be possible. 


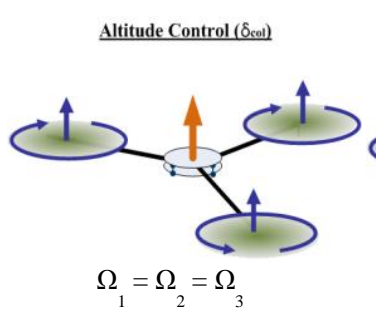

(a) (b)

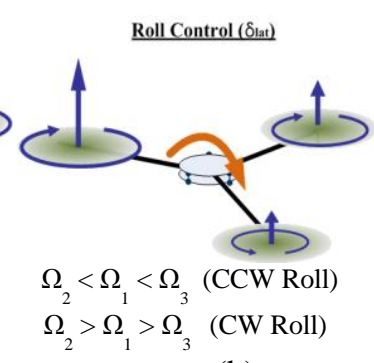

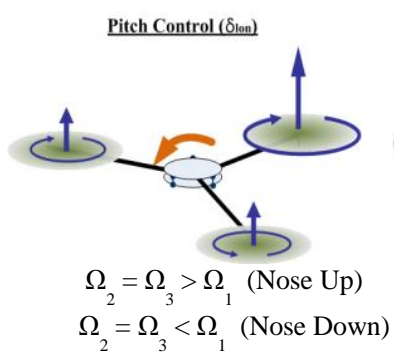

(c)

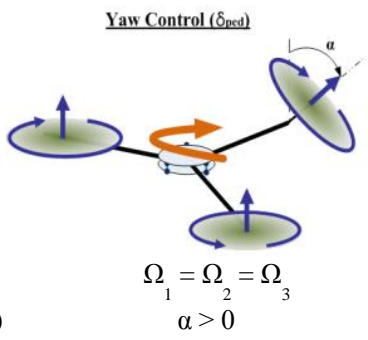

(d)
Fig. 2. Single Tri-Rotor Control Strategies (a) Altitude (b) Roll (C) Pitch (d) Yaw

\section{Control Allocation}

For a successful and stable control of Tri-Rotor UAV, an accurate control allocation is essential. Tri-Rotor will follow a control command similar to a conventional helicopter control commands, which are collective, lateral, longitudinal, and pedal [8]. They are expressed as $\delta_{\text {col }}, \delta_{\text {lat }}, \delta_{\text {lon }}, \delta_{\text {ped. }}$. Single Tri-Rotor UAV will be controlled by angular velocities of three rotors and the tilt angle of rotor 1. Figure 3 and equation (7) show the control allocation of Single Tri-Rotor.

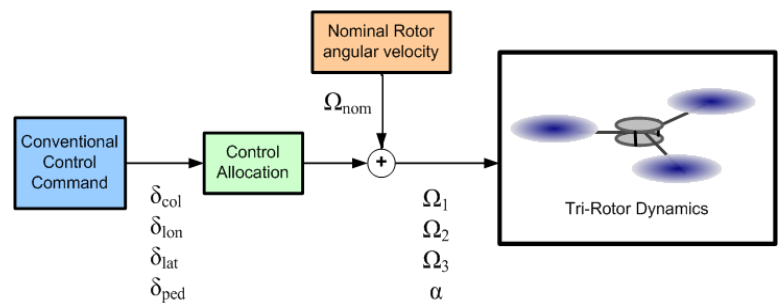

Fig. 3. Control Allocation of Tri-Rotor UAV

$$
\begin{aligned}
\Omega_{1} & =\Omega_{n o m}+\left(\delta_{c o l} / 3\right)+\left(\delta_{l o n} / 3\right) \\
\Omega_{2} & =\Omega_{n o m}+\left(\delta_{c o l} / 3\right)-\left(\delta_{l o n} / 3\right)-\left(\delta_{l a t} / 2\right) \\
\Omega_{3} & =\Omega_{n o m}+\left(\delta_{c o l} / 3\right)-\left(\delta_{l o n} / 3\right)+\left(\delta_{l o n} / 2\right) \\
\alpha & =\delta_{\text {ped }}
\end{aligned}
$$

Figure 4 shows the block diagram for attitude hold autopilot of Single Tri-Rotor UAV. The attitude hold autopilot tracks the pitch, roll, and yaw angles, and hold them. As shown in figure 4, attitude hold autopilot consists of a double-loop, which the inner-loop represents the angular rate feedback, and the outer-loop represents attitude feedback [9].

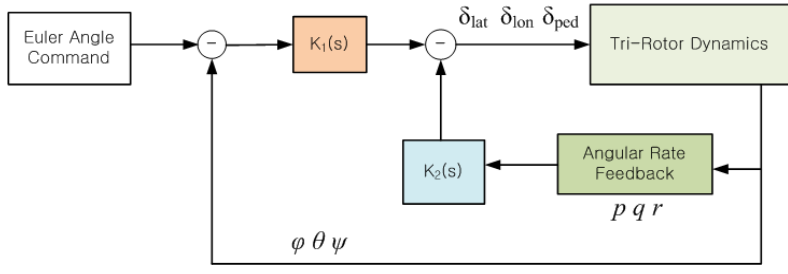

Fig. 4. Block Diagram for Attitude Hold Autopilot

In figure $4, \mathrm{~K}_{1}$ and $\mathrm{K}_{2}$ are the gain values of Euler angle attitudes and angular rates.

\section{COAXIAL TRI-ROTOR UAV}

Coaxial Tri-Rotor UAV has two rotors installed on each axis of rotation, so it has six rotors total. By having a counter rotating rotors on each axis, it is able to cancel the yawing moment on each axis. The advantage of this system is to have a better stability, but requiring more power for six rotors is the disadvantage of this system.

\section{A. Concepts and Dynamics}

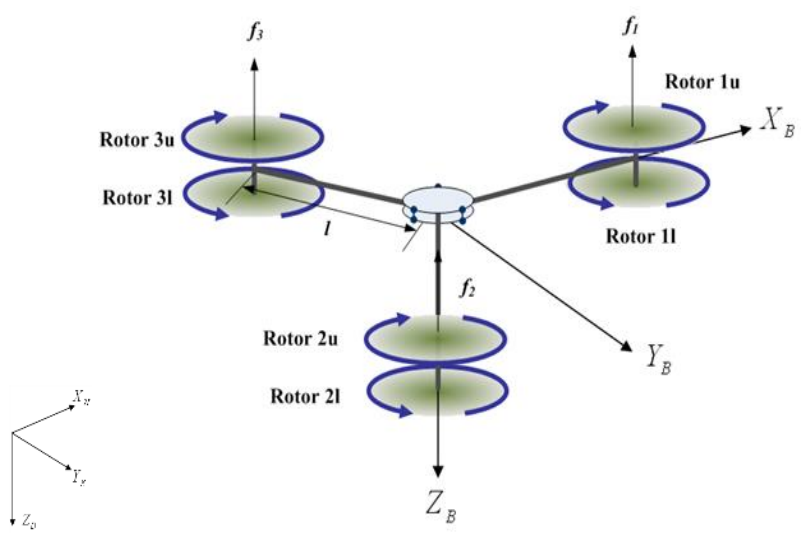

Fig. 9. Configuration of Coaxial Tri-Rotor UAV

In figure 9, Coaxial Tri-Rotor's configuration with body and reference frames are shown. As the figure shows, each axis of rotation contains upper rotors and lower rotors. All upper rotors rotate in clockwise direction, and all lower rotors rotate in counter clockwise direction. Because of this behavior, Coaxial Tri-Rotor UAV does not need an extra device, such as the servo motor used in Single Tri-Rotor, in order to cancel the yawing moment of the system. Distances from the center of the gravity to each rotor are the same and they are defined to be $l$. Forces and Moments equations were derived with respect to figure 9, and they are expressed in equations (8) and (9). As mentioned above, again, $f_{1}, f_{2}, f_{3}$ are rotor forces and $\tau_{1}, \tau_{2}, \tau_{3}$ are rotor torques, and subscripts $\mathrm{U}$ and $\mathrm{L}$ under rotor torques mean Upper and Lower respectively. 


$$
\begin{gathered}
\vec{F}=\left[\begin{array}{c}
0 \\
0 \\
-f_{1 U}-f_{1 L}-f_{2 u}-f_{2 L}-f_{3 U}-f_{3 L}
\end{array}\right] \\
\vec{M}=\left[\begin{array}{c}
\left(f_{3 U}+f_{3 L}\right) l \cos 30^{\circ}-\left(f_{2 U}+f_{2 L}\right) l \cos 30^{\circ} \\
\left(f_{1 U}+f_{1 L}\right) l-\left(f_{2 U}+f_{2 L}\right) l \sin 30^{\circ}-\left(f_{3 U}+f_{3 L}\right) l \sin 30^{\circ} \\
\tau_{1 U}+\tau_{2 U}+\tau_{3 U}-\tau_{1 L}-\tau_{2 L}-\tau_{3 L}
\end{array}\right]
\end{gathered}
$$

\section{B. Control Strategies}

Control strategies of Coaxial Tri-Rotor show a bit similar methods from those of Single Tri-Rotor UAVs. Figure 10 shows the control strategies of Coaxial Tri-Rotor UAV.

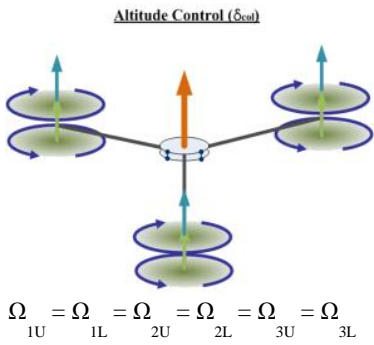

(a)

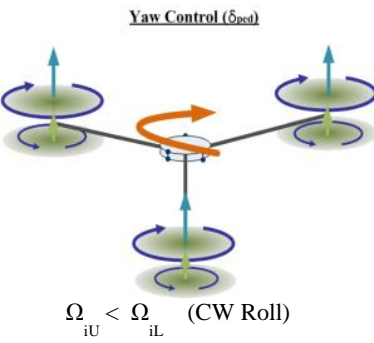

(b)

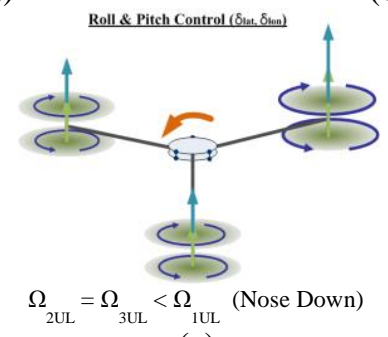

(c)

Fig. 10. Coaxial Tri-Rotor Control Strategies (a) Altitude (b) Yaw (C) Roll \& Pitch

As it is shown in figure 10, to increase the altitude, increasing all six rotor speeds at the same time will do this command. To perform a yawing control, varying the rotor speeds of upper and lower rotors will create a reaction torques, which leads to a yawing moment. Finally, roll and pitch commands could be done by increasing the upper and lower rotors of tail rotor (whichever rotor pairs located on the back) would lead to a nose down pitching moment and vice versa. Rolling is done just same as the pitching.

\section{Control Allocation}

A similar technique from Single Tri-Rotor's control system design is applied for Coaxial Tri-Rotor's control system design. There are also four command inputs allocated into six rotors. Figure 11 and equation (10) describes the control allocation of Coaxial Tri-Rotor UAV. Block diagram of Coaxial Tri-Rotor for attitude hold autopilot is similar to that of Single Tri-Rotor, which is represented in figure 4.

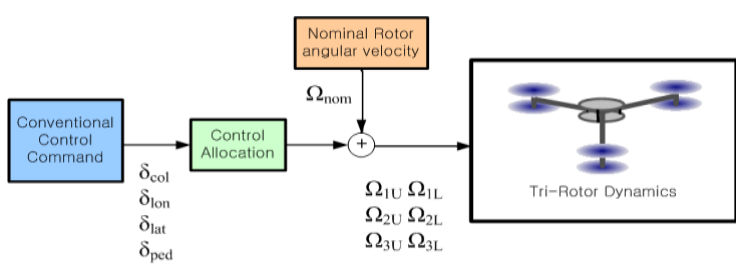

Fig. 11. Control Allocation of Coaxial Tri-Rotor UAV

$$
\begin{aligned}
& \Omega_{1 U}=\Omega_{\text {nom }}+\left(\delta_{c o l} / 6\right)+\left(\delta_{\text {lon }} / 6\right)+\left(\delta_{\text {ped }} / 6\right) \\
& \Omega_{1 L}=\Omega_{\text {nom }}+\left(\delta_{c o l} / 6\right)+\left(\delta_{\text {lon }} / 6\right)-\left(\delta_{\text {ped }} / 6\right) \\
& \Omega_{2 U}=\Omega_{n o m}+\left(\delta_{c o l} / 6\right)-\left(\delta_{\text {lon }} / 6\right)+\left(\delta_{\text {ped }} / 6\right)-\left(\delta_{\text {lat }} / 4\right) \\
& \Omega_{2 L}=\Omega_{\text {nom }}+\left(\delta_{c o l} / 6\right)-\left(\delta_{\text {lon }} / 6\right)-\left(\delta_{p e d} / 6\right)-\left(\delta_{\text {lat }} / 4\right) \\
& \Omega_{3 U}=\Omega_{\text {nom }}+\left(\delta_{c o l} / 6\right)-\left(\delta_{\text {lon }} / 6\right)+\left(\delta_{\text {ped }} / 6\right)+\left(\delta_{\text {lat }} / 4\right) \\
& \Omega_{3 L}=\Omega_{\text {nom }}+\left(\delta_{\text {col }} / 6\right)-\left(\delta_{\text {lon }} / 6\right)-\left(\delta_{\text {ped }} / 6\right)+\left(\delta_{\text {lat }} / 4\right)
\end{aligned}
$$

\section{Simulation Results}

In this section, numerical simulations for stabilization of both types of Tri-Rotor were conducted in order to observe the behaviors of UAVs in each channel with given command inputs. All simulations were done via MATLAB Simulink.

\section{A. Single Tri-Rotor Simulation Results}

Table I shows the parameters of Single Tri-Rotor that are used for the nonlinear simulations. Also, all the gain values used for this simulation and Gain Margins and Phase Margins from the Bode plots are tabulated in table II.

TABLE I

SINGLE TRI-ROTOR PARAMETERS

\begin{tabular}{cc}
\hline \hline Parameters & Values (Unit) \\
\hline Mass & $1.1(\mathrm{~kg})$ \\
$\mathrm{I}_{\mathrm{x}}$ & $0.02396\left(\mathrm{~kg} \cdot \mathrm{m}^{2}\right)$ \\
$\mathrm{I}_{\mathrm{y}}$ & $0.01271\left(\mathrm{~kg} \cdot \mathrm{m}^{2}\right)$ \\
$\mathrm{I}_{\mathrm{z}}$ & $0.01273\left(\mathrm{~kg} \cdot \mathrm{m}^{2}\right)$ \\
Length 1 & $0.2483(\mathrm{~m})$ \\
Length 2 & $0.1241(\mathrm{~m})$ \\
Length 3 & $0.2150(\mathrm{~m})$ \\
$\mathrm{K}_{\mathrm{t}}$ & $1.970 \mathrm{e}-6$ \\
$\mathrm{~K}_{\tau}$ & $2.880 \mathrm{e}-7$ \\
\hline \hline
\end{tabular}

TABLE II

Gains and Marginal Values of Single Tri-Rotor

\begin{tabular}{ccccc}
\hline \hline Channel & $\mathbf{K}_{\mathbf{1}}$ & $\mathbf{K}_{\mathbf{2}}$ & GM & PM \\
\hline Altitude & 1250 & -650 & Inf & $74.2 \mathrm{deg}$ \\
Lateral & 269.2 & 130 & Inf & $80.8 \mathrm{deg}$ \\
Longitude & 185.7 & 120 & Inf & $84.8 \mathrm{deg}$ \\
Yaw & 10.45 & 5.17 & Inf & $89.6 \mathrm{deg}$ \\
\hline \hline
\end{tabular}

Every gain margin goes to the infinity meaning every channel shows a stable behavior. Every simulation was done for five seconds. Altitude and Vertical Velocity responses are shown in figure 5, and all the angular rate responses are shown in figure 6. Figure 7 shows Euler Angle Responses, and figure 8 shows the control commands. Rising time and settling time were set between one to two seconds for each channel, and 
overshoots were set as close to zero. According to the results shown from figure 5 through 8 , it is remarkable that responses converge to zero after a short amount of time (about 1 to 2 seconds). Especially in figure 7, all the Euler angle responses show that even the initial angles were non-zeros, they converge to zero meaning that attitude controls are properly performed. In most of the plots, there are couple "bumps", and they are from the tilt angle $\alpha$ from the beginning. After about 1 to 2 seconds, plots tend to return to their original trimmed values. Therefore, these plots show that Single Tri-Rotor UAV control is possible.
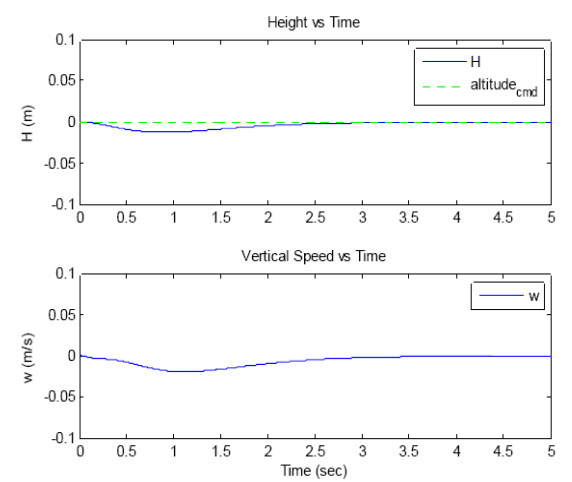

Fig. 5. Altitude and Vertical Velocity Responses
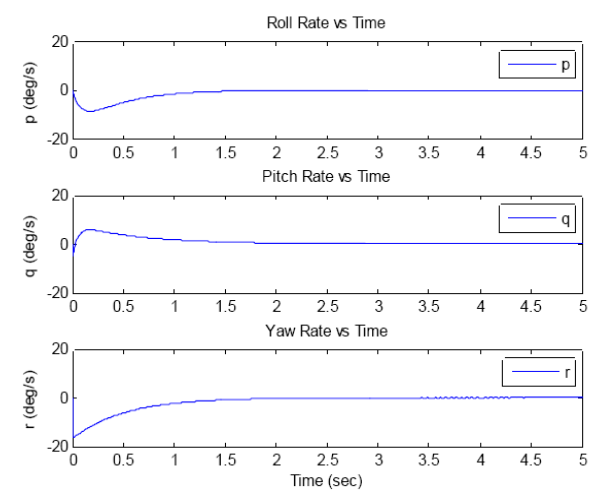

Fig. 6. Angular Rate Responses from the Body Frame
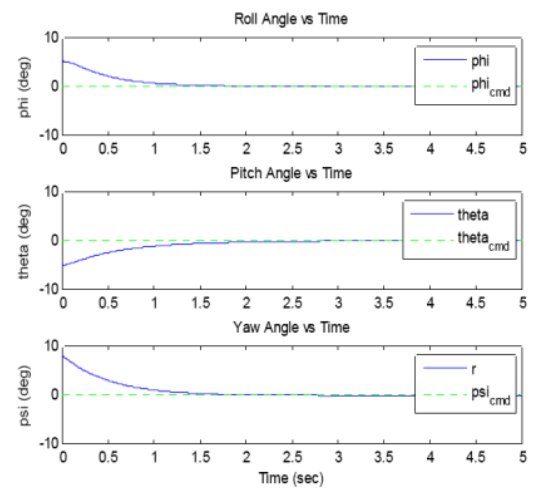

Fig. 7. Euler Angle Responses
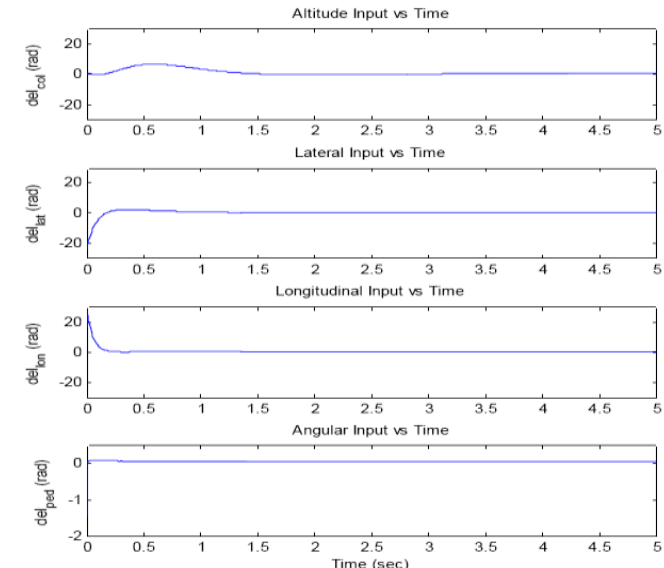

Fig. 8. Control Commands

\section{B. Coaxial Tri-Rotor Simulation Results}

For the Coaxial Tri-Rotor simulation parameters, all parameters are identical to that of Single Tri-Rotor except the fact that all lengths defined in Coaxial Tri-Rotor configuration are identical to length 1 for Single Tri-Rotor. Gain settings of Coaxial Tri-Rotor for the simulation, however, are slightly different from the gain settings of Single Tri-Rotor, and they are tabulated in table 3 as well as each channel's gain margins and phase margins.

TABLE III

Gains and Marginal Values of Coaxial Tri-Rotor

\begin{tabular}{ccccc}
\hline \hline Channel & $\mathbf{K}_{\mathbf{1}}$ & $\mathbf{K}_{\mathbf{2}}$ & $\mathbf{G M}$ & $\mathbf{P M}$ \\
\hline Altitude & 500 & 700 & Inf & $77.0 \mathrm{deg}$ \\
Lateral & 269.2 & 130 & Inf & $82.7 \mathrm{deg}$ \\
Longitude & 185.7 & 130 & Inf & $80.8 \mathrm{deg}$ \\
Yaw & 190 & 260 & Inf & $89.9 \mathrm{deg}$ \\
\hline \hline
\end{tabular}

All gains set for the simulation was aimed for the values between 1 to 3 seconds of rising time and settling time without any overshoot. Figures 9 through 12 display the simulation results of altitude and altitude rate, angular rates, and attitudes, and control commands of Coaxial Tri-Rotor UAV.
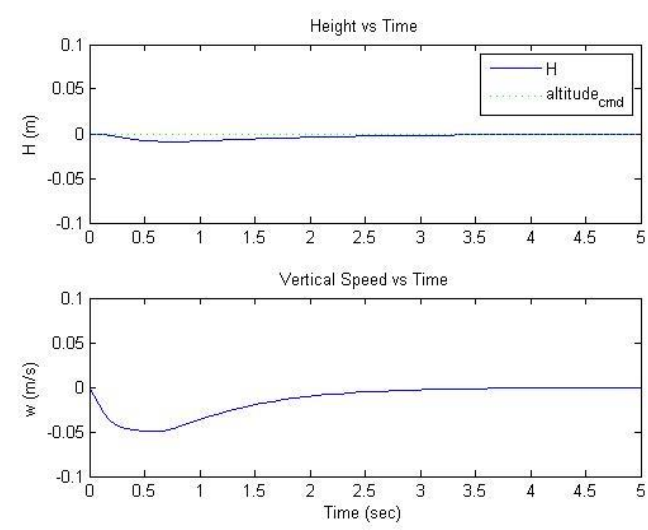

Fig. 9 Altitude and Vertical Velocity Responses 

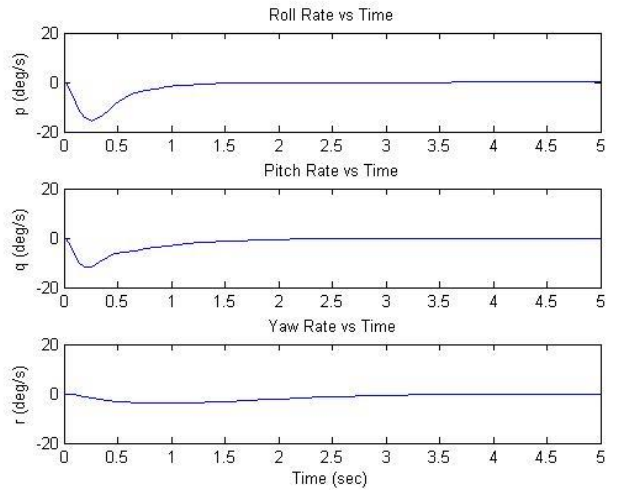

Fig. 10 Angular Rate Responses from the Body Frame
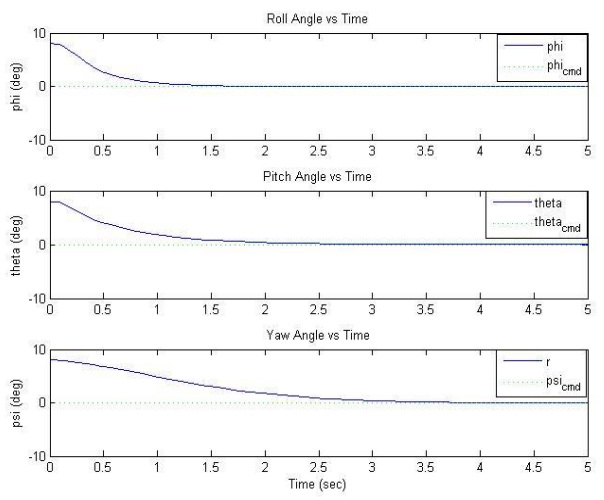

Fig. 11 Euler Angle Responses
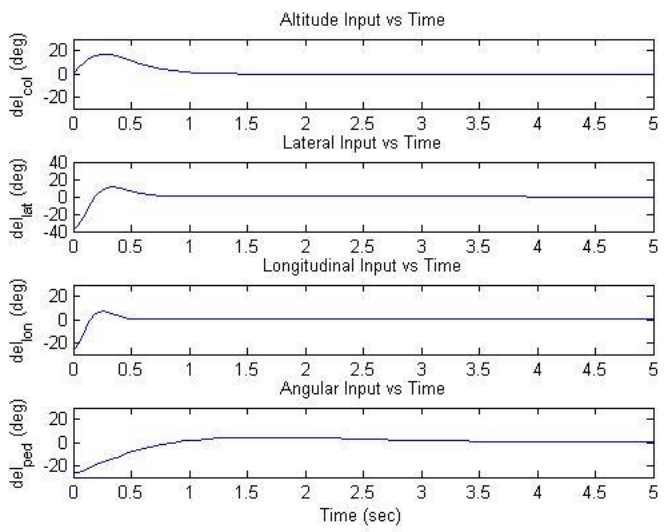

Fig. 12 Control Commands

Every simulation was performed for 5 seconds. In figures above, altitude and attitude channels successfully show their responses as originally assumed: the rising and the settling time are to be between 1 to 3 seconds with no overshoot. In figure 11, Attitude plots show that initial commands were given as eight degrees in roll, pitch, and yaw channels, and after a fair amount of time, they all converge to zero degree. These results denote that the UAV regains its stability even it has been started from an unstable position.

\section{CONCLUSION}

In this paper, the importance of a small sized VTOL Unmanned Aerial Vehicle for various missions was mentioned, and Tri-Rotor UAV was introduced as one of their examples. In order to solve the yawing moment problem that Tri-Rotor originally has, there are two types of Tri-Rotor UAV introduced in this paper: they are Single Tri-Rotor UAV and Coaxial-Tri-Rotor UAV. Single Tri-Rotor UAV solves the yawing moment problem by tilting the tail rotor by the angle $\alpha$. Coaxial Tri-Rotor solves the same problem by installing two counter rotating rotors, which cancel each other's reaction torques, on each axis. For both types of Tri-Rotor UAVs, attitude and altitude stabilizations were necessary, and nonlinear simulations were carried out in order to observe the stabilities of both UAVs. Simulation results showed that altitude and attitude responses follow the commands with reasonable rising times and settling times. After observing the simulation results, it is concluded that altitudes and attitude stabilizations for both types of Tri-Rotor are accomplished properly.

\section{ACKNOWLEDGMENT}

Authors are gratefully acknowledging the financial support by Agency for Defense Development and by UTRC (Unmanned Technology Research Center) and Brain Korea 21 Project, Korea Advanced Institute of Science and Technology.

\section{REFERENCES}

[1] A.Tayebi, S.McGilvray, "Attitude stabilization of a four-rotor aerial robot," IEEE Conference on Decision and Control, 2004

[2] N.Guenard, T.Hamel, V.Moreau, " Dynamic modeling and intuitive control strategy for an "X4-flyer," International Conference on Control and Automation, 2005

[3] S. Salazar-cruz, F. Kendoul, R. Lozano, I. Fantoni, "Real-Time Stabilization of a Small Three-Rotor Aircraft," Aerospace and Electronic Systems Vol.44, No.2, 2008

[4] J.Escareno, A.Sanchez, O.Garcia, R.Lozano, "Triple Tilting Rotor mini-UAV: Modeling and Embedded Control of the Attitude," American Control Conference, 2008

[5] S.Salazar-Cruz, R.Lozano, "Stabilization and Nonlinear Control for a novel trirotor mini-aircraft," International Conference on Robotics and Automation, 2005

[6] B.L, Stevens and F.L.Lewis, Aircraft control and simulation, Jon Wiley \& Sons, INC, 1992.

[7] B. Mettler, Identification Modeling and Characteristics of Miniature Rotorcraft, Kluwer Academic Publisher, 2003.

[8] G.D. Padfield, Helicopter Flight Dynamics: The Theory and Application of Flying Qualities and Simulation Modeling, AIAA, 1996.

[9] P.Castillo, R.Lozano, "Stabilization of a mini-rotorcraft having four rotors," IEEE/RSJ International Conference of Intelligent Robots and Systems, 2004. 